\title{
Consumo alcohólico en la población española
}

\author{
Sánchez Pardo, L. \\ Consejo Asesor del Observatorio Español sobre Drogas
}

Enviar correspondencia: Lorenzo Sánchez Pardo. Delegación del Gobierno para el Plan Nacional sobre Drogas. C/ Recoletos, 22. 28001 Madrid. Teléfono 91 5372735. Correo electrónico: Isanchezpa@nexo.es

\section{Resumen}

La masiva incorporación de adolescentes y mujeres al consumo de alcohol registrada en España en las dos últimas décadas, ha convertido a esta sustancia en la principal droga de abuso de nuestro país. La importante presencia que tienen las bebidas alcohólicas en la sociedad española queda confirmada por el hecho de que una amplia mayoría (el 87\%) de los ciudadanos de 15 a 65 años las ha consumido en alguna ocasión o porque el 4 '7\% las consume con una frecuencia semanal y un $13 \%$ diariamente.

Los resultados de las Encuestas Domiciliarias sobre Drogas, realizadas por la Delegación del Gobierno para el Plan Nacional sobre Drogas en los años 1995, 1997 y 1999, permiten constatar la quiebra del ciclo expansivo que los consumos de alcohol han registrado en España en los últimos años. Este proceso ha sido posible gracias a la estabilización registrada en las edades de inicio al consumo (tras años de constante reducción), la disminución de los niveles de experimentación (crece el número de abstemios) y la moderación de los consumos entre los varones. Pero sin duda, el fenómeno más relevante que se ha producido en este período ha sido la importante reducción de los porcentajes de bebedores abusivos y de gran riesgo, tanto entre los consumidores de alcohol en días laborables como en fin de semana.

La estabilización de los consumos de bebidas alcohólicas y la drástica reducción de los consumos de riesgo ha sido posible gracias a la creciente sensibilización de los ciudadanos respecto a los problemas asociados al consumo de alcohol. No obstante, se observa todavía la existencia de un contexto social tolerante con el consumo de bebidas alcohólicas, como lo confirma el bajo riesgo asociado al consumo de las mismas, a pesar de que la mortalidad atribuible al alcohol supera las 12.000 muertes anuales.

Palabras claves: bebidas alcohólicas, prevalencias de uso, consumos abusivos, embriagueces, percepciones sociales, mortalidad atribuible, España.

\section{Summary}

The huge number of adolescents and females who had an abusive alcohol consumption in the last two decades, has turned this substance in the first drug of abuse consumed in Spain.. The strong presence of alcohol among the Spanish society is confirmed by the fact that a great majority of the population $(87 \%)$ has consumed it at least once, or because $47 \%$ has a weekly consumption and 13\% has a daily consumption.

Data of a domiciliary survey on drugs made by The National Plan on Drugs of Spain in 1995, 1997 and 1999 shows the end of the tendency to increase of alcohol consumption registered in Spain. This process has been possible thanks to the stabilization registered on the ages of first alcohol use (after years of continuous reduction), the reduction of the experimentation levels (there is an increase of abstemious), and the moderation of alcohol consumption among males. But without any doubt, the most relevant fact that has taken place during this period, has been the relevant reduction of the percentages of heavy drinkers with risk behavior, both the ones who drink daily as well as the ones who drink on weekends.

The stabilization of alcoholic beverage consumption and the sharp reduction of risk consumption attitudes have been possible due to the increasing awareness of the population in relation to alcohol-related problems. But a permissive social context in relation to alcohol use, still can be observed as it is confirmed by the low perception of risks associated to its consumption, notwithstanding that mortality associated to alcohol abuse is over 12,000 deaths each year.

Key Words: alcoholic beverages, usage prevalence, abusive consumption, inebriety, social perceptions, mortality, Spain. 


\section{INTRODUCCIÓN}

E modelo tradicional de consumo de drogas dominante en España hasta la década de los setenta, caracterizado por el consumo de sustancias como el alcohol o el tabaco por parte de los varones adultos, contaba con una amplia legitimación social y cultural, lo que contribuyó a que la sociedad española percibiera como naturales los consumos de sustancias que ya entonces tenían un impacto social y sanitario muy negativo. Sin embargo, las relaciones de los ciudadanos con las drogas se verán profundamente alteradas en la década de los ochenta y los noventa, primero como consecuencia de la irrupción de nuevas sustancias ilícitas en el mercado y más tarde por efecto de la incorporación masiva de los jóvenes y las mujeres al consumo de las drogas.

La aparición de un nuevo modelo de consumo de drogas supone la modificación de las funciones asignadas hasta entonces al uso de estas sustancias. Lo lúdico se convierte en el objetivo básico de los consumos, sin menoscabo de otras funciones secundarias como el fortalecimiento de la integración grupal o como forma de expresión o identificación de ciertas corrientes culturales o políticas ${ }^{1}$.

Este nuevo modelo dominante de uso de las drogas ha tenido dos efectos fundamentales en el ámbito de los consumos de las bebidas alcohólicas:

a) La consolidación de dos patrones de consumo de alcohol diferenciados entre los adultos y los jóvenes. Si las relaciones con el alcohol de los adultos se caracterizan por la regularidad de su uso, la vinculación a la gastronomía y a ciertos acontecimientos sociales o el que una buena parte de la ingesta de bebidas alcohólicas se realice en el hogar; para los jóvenes el consumo se asocia principalmente con las noches del fin de semana, con la diversión y se realiza con el grupo de iguales y en espacios y lugares públicos. b) La asociación del consumo de alcohol con el de otras sustancias, más allá de su tradicional vinculación con el tabaco. El policonsumo se ha convertido en el patrón dominante de uso de drogas, un patrón, que de forma especial entre los jóvenes, tiene al alcohol como sustancia de referencia.

Pocas sustancias como el alcohol ejemplifican las relaciones, con frecuencia contradictorias y cambiantes, que establecen los ciudadanos con las drogas. El análisis de los discursos sociales dominantes en la sociedad española en torno a las drogas pone de relieve algunas de estas contradicciones: frente a la alarma social y el rechazo generalizado que genera el consumo de las drogas ilícitas existe todavía una importante tolerancia social frente a los consumos de alcohol, ante los riesgos y efectos negativos que se atribuyen al consumo de la mayor parte de las drogas ilícitas se banalizan los efectos asociados al consumo de alcohol (a pesar de la elevada morbilidad y mortalidad atribuible a los mismos) o bien se legitiman y banalizan los consumos de alcohol de la población adulta, mientras que se muestra rechazo y alarma frente a los consumos que realizan los jóvenes. Como han evidenciado investigaciones recientes ${ }^{2}$, los factores generacionales y la experiencia personal con las sustancias condicionan los discursos y las posiciones frente al alcohol y otras drogas, configurando un panorama que oscila entre la aceptación más o menos tácita de los consumos (discurso dominante entre los jóvenes) y su abierto rechazo (posición mayoritaria entre los adultos).

A pesar de que el contexto social y cultural en el que se desenvuelven las relaciones de los ciudadanos con las bebidas alcohólicas favorece el uso de las mismas, se han producido en los últimos años progresos significativos. Junto a la reducción de los niveles de experimentación con el alcohol, se constata el descenso de los bebedores diarios y, lo que sin duda es más relevante, la significativa reducción del número de bebedores abusivos y de riesgo ${ }^{3}$. De hecho, el intenso proceso de 
moderación de los patrones de consumo de bebidas alcohólicas más problemáticos es sin duda el fenómeno que mejor caracterizaría la evolución registrada por los consumos de bebidas alcohólicas en los últimos años en España. Por otra parte, la tendencia seguida por los consumos de alcohol entre los adolescentes en los últimos años ${ }^{4}$ permite cierto optimismo respecto a la evolución futura de los consumos de bebidas alcohólicas en España.

\section{EVOLUCIÓN DEL CONSUMO DE BEBI- DAS ALCOHÓLICAS EN LA POBLACIÓN ESPAÑOLA}

El Programa de Encuestas Domiciliarias sobre Drogas, que desde 1995 viene realizando con carácter bianual la Delegación del Gobierno para el Plan Nacional, permite el análisis de los hábitos de consumo de bebidas alcohólicas de la población española, así como de las opiniones y actitudes frente a los mismos. La metodología utilizada se ha mantenido estable en las encuestas realizadas en 1995, 1997 y 1999, con la salvedad de la acotación registrada en el universo poblacional en las encuestas de 1997 y 1999 (españoles de 15 a 65 años) en relación a la de 1995 (15 y más años).

El análisis de los resultados de estas encuestas, que serán descritos a continuación, permite establecer un balance general de la evolución de los consumos de alcohol en el período 1995-1999, que tendría como aspectos positivos:

- La reducción de los niveles de experimentación con el alcohol.

- La mayor sensibilización social respecto a los problemas asociados al consumo de alcohol.

- La importante reducción en el número de bebedores abusivos y en especial de los considerados como de gran riesgo.

- La estabilización de las edades de inicio al consumo tras años de constante reducción en las mismas.
Por su parte, como elementos negativos cabría señalar:

- El incremento de la continuidad o fidelización en el uso de la sustancia.

- La mayor presencia de mujeres entre la población consumidora.

Conviene destacar que esta evolución es en gran medida coincidente con las tendencias en el consumo de bebidas alcohólicas que se vienen observando en Europa. Los datos aportados en el marco de la WHO European Ministerial Conference on Young People and Alcohol (Estocolmo, 2001) ${ }^{5}$ ponen de manifiesto que los consumos per cápita de bebidas alcohólicas habrían descendido en los últimos años en la práctica totalidad de los países europeos. Por otra parte, la evolución seguida por algunos indicadores indirectos, como los casos de cirrosis por 100.000 habitantes, confirmarían la existencia en Europa de una tendencia a la reducción en el número de bebedores abusivos, similar a la apuntada para España.

\subsection{Evolución general de los consumos de alcohol}

Globalmente considerados, los distintos indicadores de uso de alcohol (consumo alguna vez en la vida, últimos 12 meses, últimos 30 días, última semana y diario) se habrían estabilizado en el período 1995-99 (Tabla 1), si bien cada uno de ellos ha seguido una tendencia diferente. Mientras se han reducido los consumos experimentales y diarios (en todos los grupos de edades), han aumentado las prevalencias de uso para los intervalos intermedios. No obstante, debe destacarse la importantísima presencia que el consumo de alcohol tiene en la sociedad española, como lo acredita que el $87 \%$ de la población de 15 a 65 lo haya probado en alguna ocasión, que cerca de la mitad lo consuma con una frecuencia semanal y que casi el $14 \%$ lo haga diariamente. 
Tabla 1. Evolución temporal de las prevalencias de consumo de alcohol (porcentaje). España, 1995-1999.

\begin{tabular}{lccc}
\hline & $\mathbf{1 9 9 5}$ & $\mathbf{1 9 9 7}$ & $\mathbf{1 9 9 9}$ \\
\hline Alguna vez en la vida & - & $89,9 \%$ & $87,1 \%$ \\
Últimos 12 meses & $68,2 \%$ & $77,8 \%$ & $74,6 \%$ \\
Últimos 30 días & $53,0 \%$ & $60,7 \%$ & $61,7 \%$ \\
Última semana & $38,9 \%$ & $48,5 \%$ & $46,7 \%$ \\
Consumo diario & $14,6 \%$ & $12,9 \%$ & $13,7 \%$ \\
\hline
\end{tabular}

Fuente: Encuestas Domiciliarias sobre Drogas 1995, 1997 y 1999. DGPNSD. Observatorio Español sobre Drogas.

\subsection{Prevalencias de consumo por sexo y grupo de edad}

a) Consumo de alcohol en los últimos 30 días

Existen diferencias en los consumos de bebidas alcohólicas en los últimos 30 días en función del sexo y la edad de los encuestados. Las prevalencias de uso continúan siendo superiores en 1999 en los hombres $\left(74^{\prime} 7 \%\right)$ que en las mujeres (50'7\%), si bien las diferencias se han reducido notablemente desde 1995 (Tabla 2).

Tabla 2. Evolución de las prevalencias de consumo en los últimos 30 días de alcohol por sexo y grupo de edad (porcentaje). España, 1995-1999.

\begin{tabular}{|c|c|c|c|c|c|c|}
\hline \multirow[b]{2}{*}{ Grupo de edad (años) } & \multicolumn{2}{|c|}{1995} & \multicolumn{2}{|c|}{1997} & \multicolumn{2}{|c|}{1999} \\
\hline & Hombres & Mujeres & Hombres & Mujeres & Hombres & Mujeres \\
\hline $15-19$ & $56,8 \%$ & $37,7 \%$ & $52,8 \%$ & $46,2 \%$ & $54,9 \%$ & $50,9 \%$ \\
\hline $20-24$ & $75,6 \%$ & $53,2 \%$ & $79,1 \%$ & $60,4 \%$ & $76,5 \%$ & $65,3 \%$ \\
\hline $25-29$ & $77,9 \%$ & $46,0 \%$ & $76,9 \%$ & $55,1 \%$ & $77,6 \%$ & $62,6 \%$ \\
\hline $30-34$ & $70,2 \%$ & $39,8 \%$ & $80,3 \%$ & $52,3 \%$ & $79,2 \%$ & $55,7 \%$ \\
\hline $35-39$ & $73,1 \%$ & $42,1 \%$ & $80,3 \%$ & $53,7 \%$ & $83,0 \%$ & $52 \%$ \\
\hline $40-65$ & $65,2 \%$ & $29,6 \%$ & $73,3 \%$ & $41,5 \%$ & $74,5 \%$ & $42,1 \%$ \\
\hline $15-65$ & $68,4 \%$ & $38,0 \%$ & $73,3 \%$ & $48,2 \%$ & $74,7 \%$ & $50,7 \%$ \\
\hline Total & \multicolumn{2}{|c|}{$53 \%$} & \multicolumn{2}{|c|}{$60,7 \%$} & \multicolumn{2}{|c|}{$61,7 \%$} \\
\hline
\end{tabular}

Fuente: Encuestas Domiciliarias sobre Drogas 1995, 1997 y 1999. DGPNSD. Observatorio Español sobre Drogas.

Entre los hombres las mayores prevalencias se concentran actualmente en el grupo de 35-39 años, con tendencia a desplazarse hacia edades superiores (en 1995 se localizaban en el grupo de 20-29 años), mientras que entre las mujeres lo hacen desde 1995 en el grupo de 20-24 años.

Destacar que todos los grupos de mujeres han registrado en el período 1995-99 un intenso incremento de los consumos de alcohol, incluido el de edad más avanzada (40-65 años), que registra una prevalencia de uso del $42 ' 1 \%$. Este incremento de la presencia femenina es más notable en los grupos más jóvenes, donde existe una práctica equiparación de hábitos entre los sexos. 
b) Consumo semanal de alcohol

El consumo de alcohol con una frecuencia semanal entre los hombres duplica al de las mujeres (62\% frente a $31^{\prime} 4 \%$ en 1999), sin que se hayan registrado variaciones relevantes desde 1995 (Tabla 3). Las mayores prevalencias de uso se localizan ente los varones en el grupo de 35-39 años y en las mujeres en el de 20-24.

Tabla 3. Evolución de las prevalencias de consumo semanal de alcohol por grupo de edad y sexo (porcentaje). España, 1995-1999.

Consumo de alcohol en la última semana.

1995

1997

1999

\begin{tabular}{|c|c|c|c|c|c|c|}
\hline \multirow[b]{2}{*}{ Grupo de edad (años) } & & & \\
\hline & Hombre & Mujer & Hombre & Mujer & Hombre & Mujer \\
\hline $15-19$ & $35,5 \%$ & $23,3 \%$ & $40,4 \%$ & $31,3 \%$ & $38,3 \%$ & $26,2 \%$ \\
\hline $20-24$ & $55,7 \%$ & $30,3 \%$ & $65,9 \%$ & $43,8 \%$ & $62,1 \%$ & $40,8 \%$ \\
\hline $25-29$ & $58,4 \%$ & $27,2 \%$ & $66,3 \%$ & $35,4 \%$ & $62,6 \%$ & $37,6 \%$ \\
\hline $30-34$ & $57,2 \%$ & $24,3 \%$ & $67,9 \%$ & $33,6 \%$ & $66,9 \%$ & $33,0 \%$ \\
\hline $35-39$ & $60,8 \%$ & $26,5 \%$ & 71,45 & $34,0 \%$ & $69,9 \%$ & $33,6 \%$ \\
\hline $40-65$ & $57,3 \%$ & $19,6 \%$ & $66,5 \%$ & $30,5 \%$ & $64,0 \%$ & $27,4 \%$ \\
\hline $15-65$ & $54,7 \%$ & $23,4 \%$ & $63,7 \%$ & $33,4 \%$ & $62,0 \%$ & $31,4 \%$ \\
\hline Total & \multicolumn{2}{|c|}{$38,9 \%$} & \multicolumn{2}{|c|}{$48,5 \%$} & \multicolumn{2}{|c|}{$46,7 \%$} \\
\hline
\end{tabular}

Fuente: Encuestas Domiciliarias sobre Drogas 1995, 1997 y 1999. DGPNSD. Observatorio Español sobre Drogas.

c) Consumo diario de alcohol

El consumo diario de alcohol continúa asociado a la población masculina adulta, como lo confirma la existencia en 1999 de un 21\% de hombres que beben diariamente frente a un $6{ }^{\prime} 2 \%$ de mujeres, sin que prácticamente se haya alterado esta relación desde 1995 (Tabla 4). Para ambos sexos las mayores consumos se localizan en el grupo de 40-65 años, con prevalencias en 1999 del 32'5\% en los hombres y del $9^{\prime} 7 \%$ en las mujeres.

En el intervalo 1995-99 todos los grupos de edades han registrado descensos en el consumo diario de alcohol, con la única excepción de las mujeres de 40-65 años, que han visto aumentar éste del 8'5\% en 1995 al 9'7\% en 1999.

Al igual que ocurre en otros indicadores, la tendencia a la equiparación de los hábitos de consumo entre los sexos que se viene observando en los grupos juveniles desde hace años provoca que, a medida que se van desplazando las cohortes generacionales en el tiempo, las mujeres adultas empiecen a tener una representación importante entre los consumidores de alcohol. Dicho de otra manera, las chicas jóvenes que en los inicios de los años ochenta comenzaron a consumir alcohol, están ya representadas en los intervalos de edades adultos ${ }^{6}$.

\subsection{Continuidad en el consumo de alcohol}

El menor grado de experimentación con las bebidas alcohólicas se compatibiliza con una mayor continuidad o fidelización en el consumo. De hecho la continuidad para los indicadores consumo en los últimos 12 meses últimos 30 días ha crecido en 6'2 puntos entre 1995 y 1999 (Tabla 5). Aunque la continuidad sigue siendo superior entre los hombres $\left(90^{\prime} 5 \%\right.$ frente a $\left.75^{\prime} 7 \%\right)$, ha aumentado desde 1995 de forma más intensa en las mujeres. Entre los hombres la mayor continuidad se registra entre los adultos (40-65 años), en tanto que en las mujeres en el grupo de 20-24 años. 
Tabla 4. Evolución de las prevalencias de consumo diario de alcohol por grupo de edad y sexo (porcentaje). España, 1995-1999.

\begin{tabular}{|c|c|c|c|c|c|c|}
\hline \multirow[b]{2}{*}{ Grupo de edad (años) } & \multicolumn{2}{|c|}{1995} & \multicolumn{2}{|c|}{1997} & \multicolumn{2}{|c|}{1999} \\
\hline & Hombre & Mujer & Hombre & Mujer & Hombre & Mujer \\
\hline $15-19$ & $2,2 \%$ & $0,5 \%$ & $0,6 \%$ & $0,3 \%$ & $1,6 \%$ & $0,1 \%$ \\
\hline $20-24$ & $5,6 \%$ & $0,9 \%$ & $3,3 \%$ & $1,0 \%$ & $3,9 \%$ & $0,5 \%$ \\
\hline $25-29$ & $13,0 \%$ & $3,2 \%$ & $10,9 \%$ & $2,3 \%$ & $10,5 \%$ & $4,1 \%$ \\
\hline 30-34 & $23,4 \%$ & $6,6 \%$ & $18,3 \%$ & $3,6 \%$ & $17,9 \%$ & $5,0 \%$ \\
\hline 35-39 & $31,5 \%$ & $8,4 \%$ & $22,6 \%$ & $6,3 \%$ & $28,8 \%$ & $6,9 \%$ \\
\hline $40-65$ & $37,5 \%$ & $8,5 \%$ & $34,4 \%$ & $9 \%$ & $32,5 \%$ & $9,7 \%$ \\
\hline $15-65$ & $23,6 \%$ & $5,8 \%$ & $20,5 \%$ & $5,4 \%$ & $21,1 \%$ & $6,2 \%$ \\
\hline Total & \multicolumn{2}{|c|}{$14,6 \%$} & \multicolumn{2}{|c|}{$12,9 \%$} & \multicolumn{2}{|c|}{$13,7 \%$} \\
\hline
\end{tabular}

Fuente: Encuestas Domiciliarias sobre Drogas 1995, 1997 y 1999. DGPNSD. Observatorio Español sobre Drogas.

Tabla 5. Evolución de la continuidad en el consumo de alcohol "últimos 12 meses últimos 30 días" (porcentaje) España, 1995-1999.

\begin{tabular}{|c|c|c|c|c|c|c|}
\hline \multicolumn{7}{|c|}{ Continuidad en el consumo de alcohol (últimos 12 meses - últimos 30 días) } \\
\hline \multirow[b]{2}{*}{ Grupo de edad (años) } & \multicolumn{2}{|c|}{1995} & \multicolumn{2}{|c|}{1997} & \multicolumn{2}{|c|}{1999} \\
\hline & Hombre & Mujer & Hombre & Mujer & Hombre & Mujer \\
\hline $15-19$ & $78,1 \%$ & $64,9 \%$ & $69,5 \%$ & $60,8 \%$ & $78,5 \%$ & $72,8 \%$ \\
\hline $20-24$ & $89,8 \%$ & $72,3 \%$ & $87,5 \%$ & $72,4 \%$ & $90,3 \%$ & $81,3 \%$ \\
\hline $25-29$ & $90,8 \%$ & $69,7 \%$ & $85,7 \%$ & $70,6 \%$ & $89,7 \%$ & $80,2 \%$ \\
\hline $30-34$ & $86,5 \%$ & $65,6 \%$ & $89,0 \%$ & $69,3 \%$ & $89,9 \%$ & $77,7 \%$ \\
\hline $35-39$ & $89,6 \%$ & $68,9 \%$ & $90,3 \%$ & $72,5 \%$ & $92,7 \%$ & $74,4 \%$ \\
\hline $40-65$ & $86,2 \%$ & $60,5 \%$ & $88,5 \%$ & $67,4 \%$ & $92,9 \%$ & $72,6 \%$ \\
\hline $15-65$ & $86,8 \%$ & $65,7 \%$ & $86,1 \%$ & $68,4 \%$ & $90,5 \%$ & $75,7 \%$ \\
\hline Total & \multicolumn{2}{|c|}{$77,7 \%$} & \multicolumn{2}{|c|}{$78,0 \%$} & \multicolumn{2}{|c|}{$83,9 \%$} \\
\hline
\end{tabular}

Fuente: Encuestas Domiciliarias sobre Drogas 1995, 1997 y 1999. DGPNSD. Observatorio Español sobre Drogas.

\subsection{Tipología de los consumidores de be- bidas alcohólicas}

El análisis de las cantidades de alcohol ingerido resulta básico para definir los perfiles de los distintos grupos de bebedores y en especial de aquellos que presentan consumos abusivos. Para facilitar la cuantificación de los consumos de bebidas alcohólicas se han elaborado distintas tipologías basadas en la utilización de una unidad de medida deno- minada UBE (Unidad Básica Estándar), que simplifica el computo de las cantidades de alcohol ingerido del siguiente modo:

-1 cerveza/vino/aperitivo = 1 UBE

-1 consumición con destilados $=2$ UBEs

Existen diversas clasificaciones, en función del número de categorías que incorporan y los umbrales establecidos para situar los niveles de abuso de alcohol. La Organización Mundial de la Salud (OMS) establece tres 
tipos de bebedores en base al consumo semanal de alcohol:

- Abstemio: ningún consumo

- Normativo: hasta 280 gr./semana (28 UBEs) en los varones y hasta 168 gr./semana (17 UBEs) en las mujeres.

- De riesgo: más de 280 gr./semana en varones y de $168 \mathrm{gr}$./semana en mujeres.
La utilización de estos criterios, a partir del registro del consumo semanal de alcohol entre las personas que afirman haber ingerido alcohol en los 30 días previos a la realización de la Encuesta Domiciliaria de 1999 (Tabla 6), permite constatar que el 9'5\% de la población española de 15 a 65 años serían bebedores de riesgo. El porcentaje de bebedores de riesgo entre los hombres duplicaría casi al de mujeres.

\section{Tabla 6. Tipología de bebedores en función del consumo medio semanal de alcohol entre quienes han consumido alcohol en los últimos $\mathbf{3 0}$ días, según criterios de la OMS (porcentaje). España, 1999.}

\begin{tabular}{lccc}
\hline & & \multicolumn{2}{c}{ Tipo de bebedor } \\
\cline { 3 - 4 } Sexo & Abstemio & Normativo & Riesgo \\
\hline Hombre & $25,8 \%$ & $62,0 \%$ & $12,1 \%$ \\
Mujer & $49,9 \%$ & $43,2 \%$ & $6,9 \%$ \\
Total & $37,9 \%$ & $52,6 \%$ & $9,5 \%$ \\
\hline
\end{tabular}

Fuente: Encuesta Domiciliaria sobre Drogas 1999. DGPNSD. Observatorio Español sobre Drogas.

Por su parte la Dirección General de Salud Pública y Consumo ( $\mathrm{M}^{\circ}$ de Sanidad y Consumo) utiliza una tipología, elaborada a partir de los consumos medios diarios de alcohol, que incorpora seis categorías de bebedores:

Tipología de bebedores establecida por la Dirección General de Salud Pública del Ministerio de Sanidad y Consumo en función del consumo diario medio de alcohol.

\begin{tabular}{lcccccccc}
\hline & \multicolumn{3}{c}{ Hombres } & & \multicolumn{3}{c}{ Mujeres } \\
\cline { 2 - 3 } Tipo de bebedores & MI/cc & Gr & UBEs & & MI/cc & Gr & UBEs \\
\hline 1. Abstinentes & 0 & 0 & 0 & & 0 & 0 & 0 \\
2. Ligeros & $12,5-25$ & $0,8-20$ & $1-2$ & & $1-25$ & $0,8-20$ & $1-2$ \\
3. Moderados & $26-75$ & $21-60$ & $3-6$ & & $26-50$ & $21-40$ & $3-4$ \\
4. Altos & $76-100$ & $61-80$ & $7-8$ & & $51-75$ & $41-60$ & $5-6$ \\
5. Excesivos & $101-150$ & $81-120$ & $9-12$ & & $76-100$ & $61-80$ & $7-8$ \\
6. Gran riesgo & +150 & +120 & +13 & & +100 & +80 & +8 \\
\hline
\end{tabular}

Fuente: Dirección General de salud Pública y Consumo. Ministerio de Sanidad y Consumo.

La aplicación de esta tipología a los resultados de las Encuestas Domiciliarias sobre Drogas de 1997 y 1999, sobre la base de los consumos de alcohol realizados por los encuestados en los últimos 30 días, de forma independiente según se trate de días laborables o de fin de semana, ofrece los siguientes resultados: 
A) Tipología de bebedores en días laborables

Casi la mitad de las personas que han bebido alcohol en los últimos 30 días han permanecido abstinentes durante los días laborables (46\%), hecho más frecuente entre las mujeres (casi 6 de cada 10). Por su parte, cerca del $30 \%$ de los encuestados serían bebedores ligeros y un $18,1 \%$ moderados.
Las restantes categorías agrupan a bebedores que realizan consumos considerados como abusivos (altos, excesivos y de gran riesgo), entre las cuales las presencia de los varones es superior. No obstante, entre los bebedores de gran riesgo (aquellos con mayores niveles de ingesta de alcohol) las diferencias entre los sexos se acortan notablemente, siendo sólo superiores en cuatro décimas para los hombres (Tabla 7).

Tabla 7. Tipología de bebedores en días laborables en función del consumo diario medio de alcohol (entre quienes han consumido alcohol en los últimos $\mathbf{3 0}$ días) por sexo y grupo edad (porcentaje y número estimado). España, 1999.

\begin{tabular}{|c|c|c|c|c|c|c|c|c|c|}
\hline & \multirow{2}{*}{$\begin{array}{c}\mathbf{N}^{\circ} \\
\text { (Miles) }\end{array}$} & \multirow[b]{2}{*}{$\%$} & \multirow[b]{2}{*}{ Hombre } & \multicolumn{6}{|c|}{ Grupo de edad (años) } \\
\hline & & & & $15-19$ & $20-24$ & $25-29$ & $30-34$ & $35-39$ & $40-65$ \\
\hline Cero UBE & 7.863 & $46 \%$ & $36,6 \% 59,8 \%$ & $78,3 \%$ & $67,6 \%$ & 56,85 & $47,4 \%$ & 38,6 & $30,3 \%$ \\
\hline Ligero (1-2 UBES) & 5.105 & $29,9 \%$ & $30,3 \% 29,1 \%$ & $10,8 \%$ & $16,6 \%$ & $21,5 \%$ & $28,4 \%$ & 33,9 & 40,2 \\
\hline Moderado (3-6/3-4 UBES) & 3.096 & $18,1 \%$ & $25,5 \% \quad 7,3 \%$ & $6,6 \%$ & $10,1 \%$ & $14,5 \%$ & $18,0 \%$ & $21,7 \%$ & $23,3 \%$ \\
\hline Alto (7-8/5-6 UBES) & 430 & $2,5 \%$ & $3,0 \% \quad 1,8 \%$ & $1,0 \%$ & $2,4 \%$ & $3,6 \%$ & $1,7 \%$ & $2,7 \%$ & $2,7 \%$ \\
\hline Excesivo (9-12/7-8 UBES) & 342 & $2 \%$ & $2,9 \% \quad 0,6 \%$ & $1,9 \%$ & $1,8 \%$ & $2,0 \%$ & $1,8 \%$ & $1,6 \%$ & $2,3 \%$ \\
\hline Gran riesgo $(+13 /+8$ UBES) & 263 & $1,5 \%$ & $1,7 \% \quad 1,3 \%$ & $1,4 \%$ & $1,6 \%$ & $1,7 \%$ & $2,6 \%$ & $1,5 \%$ & $1,2 \%$ \\
\hline Total & 17.106 & & & & & & & & \\
\hline \multicolumn{2}{|c|}{ Alto+Excesivo+Gran Riesgo } & $6 \%$ & $3,7 \%$ & $4,3 \%$ & $5,8 \%$ & $7,3 \%$ & 6,1 & 5,8 & 6,2 \\
\hline
\end{tabular}

Fuente: Encuesta Domiciliaria sobre Drogas 1999. DGPNSD. Observatorio Español sobre Drogas

El análisis de los consumos abusivos de alcohol en los días laborables permite extraer algunos datos relevantes:

- El 6\% de la población española de 15 a 65 años (algo más de 1 millón de personas) mantiene un patrón de consumo abusivo de alcohol en los días laborables (Alto+Excesivo+Gran Riesgo).

- Un 1'5\% de los encuestados (unas 263.000 personas) son bebedores de gran riesgo.

- El patrón de consumo abusivo de alcohol en días laborables es más evidente entre los hombres $\left(7^{\prime} 6 \%\right.$ frente al $3^{\prime} 7 \%$ de las mujeres) y en los grupos de $25-29$ y $40-$ 65 años.

- Los varones de 30-34 años y las mujeres de 25-29 son los colectivos con mayor prevalencia de consumos abusivos de alcohol (Tabla 8).

- Los mayores porcentajes de bebedores de gran riesgo se localizan entre los varones de 30-34 años. Por su parte, entre las mujeres se concentran en el grupo de edades muy jóvenes (15-29 años), hecho especialmente preocupante.

El hábito de no consumir bebidas alcohólicas durante los días laborables está más arraigado en el grupo de 15-19 años, de forma más intensa entre las mujeres $(85,1 \%)$ que entre los hombres $(72,4 \%)$.

La evolución de las distintas categorías de bebedores en días laborables en el período 1997-99 permite extraer algunas conclusiones interesantes:

- El porcentaje de población que habiendo consumido alcohol en los últimos 30 días 
Tabla 8. Tipología de bebedores en días laborables en función del consumo diario medio de alcohol (entre quienes han consumido alcohol en los últimos $\mathbf{3 0}$ días) por sexo y grupo de edad simultáneamente (porcentaje). España, 1999.

\begin{tabular}{lcccccccccccc}
\hline & \multicolumn{10}{c}{ Grupo de edad (años) } \\
\cline { 2 - 13 } & \multicolumn{2}{c}{$\mathbf{1 5 - 1 9}$} & $\mathbf{2 0 - 2 4}$ & $\mathbf{2 5 - 2 9}$ & $\mathbf{3 0 - 3 4}$ & $\mathbf{3 5 - 3 9}$ & $\mathbf{4 0 - 6 5}$ \\
\cline { 2 - 13 } & Hombre & Mujer & Hombre & Mujer & Hombre & Mujer & Hombre & Mujer & Hombre & Mujer & Hombre & Mujer \\
\hline Cero UBE & 72,4 & 85,1 & 58,3 & 79,0 & 49,7 & 65,9 & 38,4 & 60,6 & 31,6 & 49,9 & 21,1 & 45,9 \\
Ligero & 12,5 & 8,9 & 18,7 & 14,0 & 22,6 & 20,0 & 27,4 & 29,8 & 32,2 & 36,7 & 39,4 & 41,6 \\
Moderado & 9,7 & 3,2 & 15,7 & 3,2 & 19,6 & 7,9 & 25,3 & 7,3 & 29,1 & 9,8 & 31,8 & 8,9 \\
Alto & 1,4 & 0,4 & 29,9 & 1,6 & 3,0 & 4,2 & 1,7 & 1,7 & 3,7 & 1,0 & 3,4 & 1,6 \\
Excesivo & 3,0 & 0,6 & 2,9 & 0,3 & 3,3 & 0,3 & 2,8 & 0,4 & 1,9 & 1,1 & 3,1 & 0,8 \\
Gran riesgo & 1,0 & 1,8 & 1,4 & 1,8 & 1,7 & 1,7 & 4,3 & 0,2 & 1,5 & 1,5 & 1,2 & 1,2 \\
Alto+excesivo+ & & & & & & & & & & & & \\
Gran riesgo & 5,4 & 2,8 & 7,2 & 3,7 & 8,0 & 6,2 & 8,8 & 2,3 & 7,1 & 3,6 & 7,7 & 3,6 \\
\hline
\end{tabular}

Fuente: Encuesta Domiciliaria sobre Drogas 1999. DGPNSD. Observatorio Español sobre Drogas.

se mantuvo abstinente los días laborables creció ligeramente entre 1997 y 1999 (del 44,5\% al 46\%), en idénticas proporciones para ambos sexos

- A pesar de las elevadas prevalencias que todavía registran los consumos abusivos de alcohol, se constata un dato altamente positivo como es la importantísima reducción de los bebedores abusivos (altos+excesivos+ gran riesgo), que pasaron del 9,9\% en 1997 al 6\% en 1999 (cerca de 460.000 españoles habrían aban- donado el consumo abusivo de alcohol). Este proceso se produce en ambos sexos.

- Especial valor adquiere la reducción de los bebedores de gran riesgo, que se habrían reducido en 1999 hasta situarse en un tercio del nivel existente en 1997 (desde el $4,4 \%$ al 1,5\%). Esta reducción, aunque perceptible en ambos sexos, ha sido mucho más intensa entre los hombres, al pasar el porcentaje de bebedores de gran riesgo del $5,7 \%$ al $1,7 \%$ (Tabla 9 y Figura 1).

Tabla 9. Evolución de la tipología de bebedores en días laborables en función del consumo diario medio de alcohol (entre quienes han consumido alcohol en los últimos 30 días) por sexo (porcentaje y número estimado). España, 1997-1999.

\begin{tabular}{lcccccccc}
\hline & \multicolumn{2}{c}{$\mathbf{N}^{\mathbf{0}}$ (Miles) } & \multicolumn{2}{c}{$\%$} & \multicolumn{2}{c}{ Hombre } & \multicolumn{2}{c}{ Mujer } \\
\hline & $\mathbf{1 9 9 7}$ & $\mathbf{1 9 9 9}$ & $\mathbf{1 9 9 7}$ & $\mathbf{1 9 9 9}$ & $\mathbf{1 9 9 7}$ & $\mathbf{1 9 9 9}$ & $\mathbf{1 9 9 7}$ & $\mathbf{1 9 9 9}$ \\
\hline Cero UBE & 6.741 & 7.863 & 44,5 & 46 & 35,4 & 36,6 & 58,5 & 59,8 \\
Ligeros (1-2 UBES) & 4.568 & 5.105 & 30,2 & 29,9 & 31,1 & 30,3 & 28,7 & 29,1 \\
Moderados (3-6/3-4 UBES) & 2.345 & 3.096 & 15,5 & 18,1 & 21,0 & 25,5 & 6,9 & 7,3 \\
Altos (7-8/5-6 UBES) & 438 & 430 & 2,9 & 2,5 & 3,3 & 3 & 2,3 & 1,8 \\
Excesivos (9-12/7-8 UBES) & 388 & 342 & 2,6 & 2 & 3,5 & 2,9 & 1,1 & 0,6 \\
Gran riesgo (+13/+8 UBES) & 670 & 263 & 4,4 & 1,5 & 5,7 & 1,7 & 2,5 & 1,3 \\
Total & 15.152 & 17.106 & 100 & & & & & \\
Altos+Excesivos+Gran Riesgo & & & 9,9 & 6 & 12,5 & 7,6 & 5,9 & 3,7 \\
\hline
\end{tabular}

Fuente: Encuestas Domiciliarias sobre drogas 1997 y 1999. DGPNSD. Observatorio Español sobre Drogas . 


\section{Figura 1. Bebedores en días laborables en función del consumo diario de alcohol por}

sexo (consumo de alcohol en los últimos 30 días) (porcentajes). España, 1997-1999.

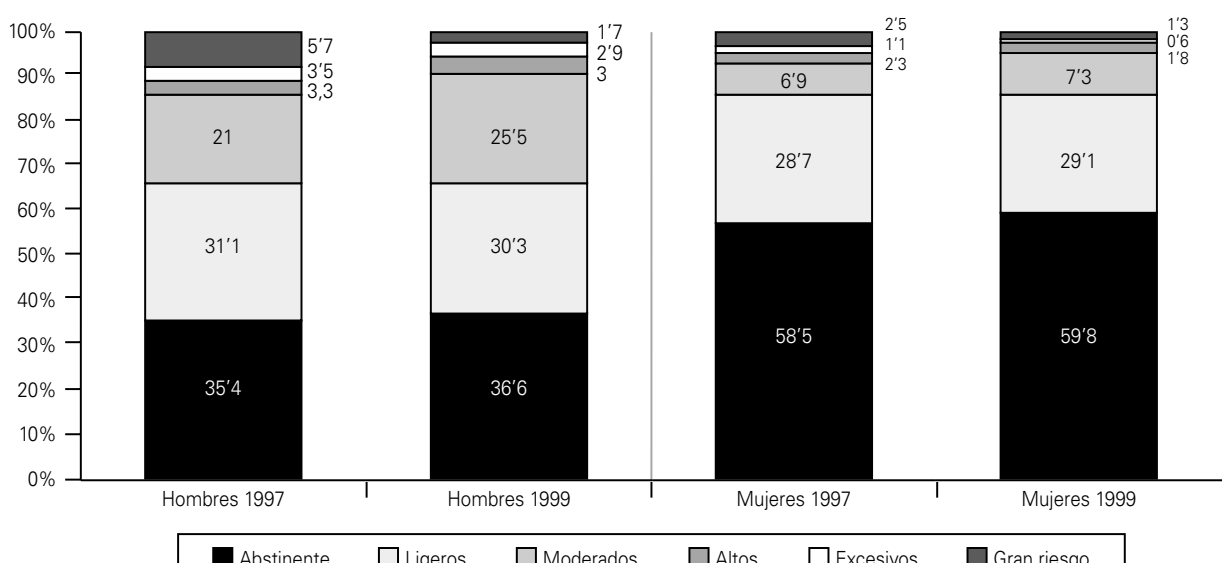

Tipología de bebedores para hombres $(\mathrm{H})$ y mujeres $(\mathrm{M})$ establecida por la Dirección General de Salud Pública. Ministerio de Sanidad y Consumo: (1 UBE equivale a 12'5-25 M/cc o a 0'8-20 gr. de alcohol)

Abstienente $0 \mathrm{H} / \mathrm{M}$ UBEs

Bebedor alto 7-8 H/5-6 M UBEs
Bebedor ligero 1-2 H/M UBEs

Bebedor excesivo 9-12 H/ 7-8 M UBEs
Bebedor moderado 3-6 H/ 3-4 M UBEs

Bebedor de gran riesgo $>13 \mathrm{H} />8 \mathrm{M}$ UBES

Fuente: DGPNSD. Observatorio Español sobre Drogas. Encuestas Domiciliarias sobre Drogas 1997-1999.

B) Tipología de bebedores en fin de semana

Los consumidores de alcohol en los últimos 30 días que no beben durante el fin de semana suponen únicamente un $8,3 \%$, si bien han aumentado con respecto a 1997 $(3,2 \%)$. En paralelo se han incrementado los bebedores ligeros, categoría que aglutina a 6 de cada 10 personas que beben en el fin de semana. Por su parte el $24,8 \%$ de los encuestados realiza consumos moderados de alcohol en el fin de semana (el 14,6\% entre las mujeres y el $31,7 \%$ entre los hombres).

El análisis de los consumos abusivos de alcohol (altos + excesivos + gran riesgo) en el fin de semana permite obtener las siguientes conclusiones:

- Un 7’7\% de los encuestados $(1.300 .000$ personas aproximadamente) tienen un patrón de consumo abusivo (Tabla 10).
- El 1,7\% de los encuestados (unas 285.000 personas) realiza consumos de gran riesgo.

- El consumo abusivo de alcohol en el fin de semana está más equilibrado entre los sexos que el observado en los días laborables, aunque sigue siendo ligeramente superior entre los hombres (8'4\% frente a $\left.6{ }^{\prime} 5 \%\right)$. Este patrón tiene sus mayores prevalencias entre los grupos de edades de 20-24 años (16'2\%) y 25-29 años (12'2\%).

- También los consumos de gran riesgo en el fin de semana son ligeramente superiores entre los hombres (un 1,8\% frente al $1{ }^{\prime} 4 \%$ de las mujeres).

- El grupo de 20-24 años, tanto para hombres como para mujeres, es el que concentra los mayores porcentajes de consumidores abusivos de alcohol (Tabla 11). 
Tabla 10. Tipología de bebedores en fin de semana en función del consumo diario medio de alcohol (entre quienes han consumido alcohol en los últimos $\mathbf{3 0}$ días) por sexo y grupo edad (porcentajes y número estimado). España, 1999.

\begin{tabular}{|c|c|c|c|c|c|c|c|c|c|}
\hline & \multirow{2}{*}{$\begin{array}{c}\mathrm{N}^{\circ} \text { (miles) } \\
\text { (miles) }\end{array}$} & \multirow[b]{2}{*}{$\%$} & \multirow[b]{2}{*}{ Hombres } & \multirow[b]{2}{*}{ Mujeres } & \multicolumn{5}{|c|}{ Grupo de edad (años) } \\
\hline & & & & & $15-19$ & 20-24 & $25-29$ & $30-34$ & $35-3940-65$ \\
\hline Cero UBE & & 8,3 & & 10,5 & & 7,8 & 7,2 & 7,5 & $9,4 \quad 8,5$ \\
\hline 2 UBES) & 10.122 & 59,2 & & 68 & & 43,6 & 48,3 & 59,4 & $61,169,2$ \\
\hline lo (3-6/3-4 UBES) & 4.244 & 24,8 & 31 & 14,6 & 28,5 & 32,5 & 32,3 & 24,8 & $25,0 \quad 18,9$ \\
\hline Alto (7-8/5-6 UBES) & 685 & 4,0 & 4,1 & 3,8 & 6,0 & 6,8 & 5,6 & 5,7 & $2,6 \quad 2,0$ \\
\hline Excesivo (9-12/7-8 UBES) & 349 & 2,0 & 2,5 & 1,3 & 3,5 & 4,4 & 3,8 & 1,1 & 1,4 \\
\hline Gran riesgo $(+13 /+8$ UBES $)$ & 285 & 1,7 & 1,8 & 1,4 & 2,3 & 5,0 & 2,8 & 1,5 & 0,5 \\
\hline Total & 17.106 & & & & & & & & \\
\hline \multicolumn{2}{|c|}{ Alto+Excesivo+Gran Riesgo } & 7,7 & 8,4 & 6,5 & 11,8 & 16,2 & 12,2 & 8,3 & 4,5 \\
\hline
\end{tabular}

Fuente: Encuesta Domiciliaria sobre Drogas 1999. DGPNSD. Observatorio Español sobre Drogas.

Tabla 11. Tipología de bebedores en fin de semana en función del consumo diario medio de alcohol en ese intervalo por sexo y grupo de edad simultáneamente (porcentaje). España, 1999.

\begin{tabular}{|c|c|c|c|c|c|c|c|c|c|c|c|c|}
\hline & \multicolumn{12}{|c|}{ Grupo de edad (años) } \\
\hline & \multicolumn{2}{|c|}{$15-19$} & \multicolumn{2}{|c|}{$20-24$} & \multicolumn{2}{|c|}{$25-29$} & \multicolumn{2}{|c|}{$30-34$} & \multicolumn{2}{|c|}{$35-39$} & \multicolumn{2}{|c|}{$40-65$} \\
\hline & Hombre & Mujer & Hombre & Mujer & Hombre & Mujer & Hombre & Mujer & Hombre & Mujer & Hombre & Mujer \\
\hline Cero UBE & 7,8 & 11,4 & 7,4 & 8,4 & 4,1 & 11,0 & 6,2 & 9,3 & 7,5 & 12,3 & 7,2 & 10,7 \\
\hline Ligero & 44,9 & 56,2 & 32,8 & 56,8 & 40,9 & 57,8 & 49,0 & 74,7 & 54,5 & 71,6 & 64,5 & 77,1 \\
\hline Moderado & 34,9 & 21,3 & 41,7 & 21,1 & 41,0 & 21,1 & 33,6 & 12,1 & 33,2 & 11,9 & 24,5 & 9,4 \\
\hline Alto & 6,6 & 5,3 & 5,6 & 8,2 & 5,1 & 6,3 & 8,4 & 1,7 & 2,7 & 2,6 & 2,2 & 1,7 \\
\hline Excesivo & 3,7 & 3,2 & 6,3 & 2,1 & 5,8 & 1,2 & 0,9 & 1,3 & 1,7 & 0,9 & 1,0 & 0,7 \\
\hline $\begin{array}{l}\text { Gran riesgo } \\
\text { Alto+excesivo+ }\end{array}$ & 2,1 & 2,6 & 6,3 & 3,4 & 3,1 & 2,5 & 1,9 & 0,9 & 0,4 & 0,7 & 0,5 & 0,4 \\
\hline gran riesgo & 12,4 & 11,1 & 18,2 & 13,7 & 14,0 & 10,0 & 11,2 & 3,9 & 4,8 & 4,2 & 3,7 & 2,8 \\
\hline
\end{tabular}

Fuente: Encuesta Domiciliaria sobre Drogas 1999. DGPNSD. Observatorio Español sobre Drogas.

Las mayores proporciones de abstinentes durante el fin de semana se registran en el grupo de 35-39 años. Por su parte, el consumo de gran riesgo alcanza sus máximas prevalencias en el grupo de edad 20-24 años, tanto para hombres $\left(6{ }^{\prime} 3 \%\right)$ como para mujeres $(3,4 \%)$.

La evolución de las distintas categorías de bebedores en el fin de semana a lo largo del período 1997-99 indica que:
- Las personas, de ambos sexos, que habiendo bebido en los últimos 30 días se mantuvo abstinente los fines de semana crecieron notablemente (del 3,2\% al 8,3\%).

- En paralelo aumentan los bebedores ligeros, aunque sólo entre los hombres.

- Las restantes categorías (bebedores moderados, altos, excesivos y de gran riesgo) han visto reducidas, para ambos sexos, sus prevalencias (Tabla 12 y Figura 2). 
Tabla 12. Evolución de la tipología de bebedores en fin de semana en función del consumo diario de alcohol (entre quienes han consumido alcohol en los últimos 30 días) por sexo (porcentaje y número estimado) España, 1997-1999.

\begin{tabular}{|c|c|c|c|c|c|c|c|c|}
\hline & \multicolumn{2}{|c|}{$N^{\circ}$ (Miles) } & \multicolumn{2}{|c|}{$\%$} & \multicolumn{2}{|c|}{ Hombre } & \multicolumn{2}{|c|}{ Mujer } \\
\hline & 1997 & 1999 & 1997 & 1999 & 1997 & 1999 & 1997 & 1999 \\
\hline Cero UBE & 477 & 1.418 & 3,2 & 8,3 & 2,7 & 6,8 & 3,8 & 10,5 \\
\hline Ligero (1-2 UBES) & 8.854 & 10.122 & 58,4 & 59,2 & 49,5 & 53 & 72,3 & 68,3 \\
\hline Moderado (3-6/3-4 UBES) & 4.140 & 4.244 & 27,3 & 24,8 & 35,2 & 31,7 & 15,2 & 14,6 \\
\hline Alto (7-8/5-6 UBES) & 720 & 685 & 4,8 & 4,0 & 5,0 & 4,1 & 4,3 & 3,8 \\
\hline Excesivo (9-12/7-8 UBES) & 512 & 349 & 3,4 & 2,0 & 4,0 & 2,5 & 2,4 & 1,3 \\
\hline Gran riesgo $(+13 /+8 U \mathrm{UES})$ & 445 & 285 & 2,9 & 1,7 & 3,5 & 1,8 & 2,1 & 1,4 \\
\hline Total & 15.152 & 17.106 & & & & & & \\
\hline Alto+Excesivo+Gran Riesgo & & & 10,1 & 7,7 & 12,5 & 8,4 & 8,8 & 6,5 \\
\hline
\end{tabular}

Fuente: Encuestas Domiciliarias sobre Drogas 1997-99. DGPNSD. Observatorio Español sobre Drogas.

Figura 2. Bebedores en fin de semana en función del consumo diario de alcohol por sexo (consumo de alcohol en los últimos 30 días) (porcentaje). España, 1997-1999.

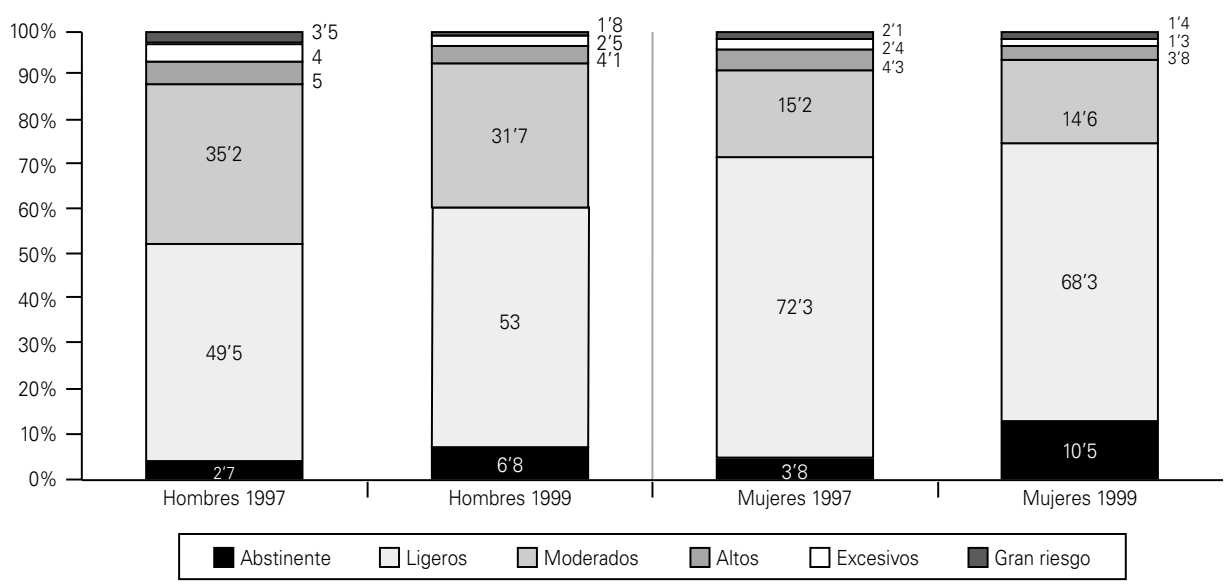

Tipología de bebedores para hombres (H) y mujeres (M) establecida por la Dirección General de Salud Pública. Ministerio de Sanidad y Consumo: (1 UBE equivale a 12'5-25 M/cc o a 0'8-20 gr. de alcohol)

Abstienente $0 \mathrm{H} / \mathrm{M}$ UBEs

Bebedor alto 7-8 H/5-6 M UBEs
Bebedor ligero 1-2 H/M UBEs

Bebedor excesivo 9-12 H/ 7-8 M UBEs
Bebedor moderado 3-6 H/ 3-4 M UBEs

Bebedor de gran riesgo $>13 \mathrm{H} />8 \mathrm{M}$ UBEs
- Con respecto a 1999 se han reducido notablemente los bebedores abusivos en los dos sexos, pasando del $10^{\prime} 1 \%$ registrado en 1997 al 7’7\% de 1999.
- Los bebedores de gran riesgo en el fin de semana también habrían registrado una reducción drástica en este período (del 2'9\% al 1'7\%). 
A modo de conclusión, destacar la existencia de un proceso de importante moderación en las cantidades de alcohol ingerido, tanto en los días laborables como en el fin de semana, habiendo sido los consumos más problemáticos los que han registrado una evolución más positiva.

\subsection{Indicadores indirectos de abuso de al- cohol}

La frecuencia de los episodios de intoxicaciones etílicas constituye un valioso indicador indirecto de los problemas de abuso con el alcohol. Los datos aportados por la Encuesta Domiciliaria sobre Drogas 1999 confirman que un elevado porcentaje de ciudadanos ha sufrido intoxicaciones etílicas en períodos recientes de su vida (Tabla 13): un 18'6\% (unos 5 millones de personas) afirma haberse emborrachado al menos en una ocasión en el último año, un 3’9\% se emborrachó con una frecuencia de al menos una vez al mes, un 1 '3\% se emborrachó con una frecuencia semanal ( 1 a 6 días a la semana) y un 0’4\% (unas 110.000 personas) se emborrachó diariamente durante el último año.

A pesar de la importante prevalencia de las intoxicaciones etílicas, hay que destacar que la evolución de las mismas en el período 1997-99 ha sido positiva, como lo pone de manifiesto el que el porcentaje de quienes se han emborrachado todos los días a lo largo de los últimos 12 meses haya caído del 1,1\% registrado en 1997 al 0,4\% de 1999. Apuntar que en este intervalo se han reducido de manera sustancial las borracheras con frecuencias más intensas (diarias, semanales y mensuales), cuyo porcentaje acumulado habría pasado del 5,3\% al 3,9\%. La reducción de las intoxicaciones etílicas, tanto para las frecuencias diarias, semanales y mensuales, observada entre 1997-99 se ha producido en ambos sexos.

Tabla 13. Evolución de las intoxicaciones etílicas entre quienes se han emborrachado en los últimos 12 meses (número estimado de personas y porcentaje). España, 1997-1999.

\begin{tabular}{lcccccc}
\hline & \multicolumn{2}{c}{$\mathbf{1 9 9 7}$} & & \multicolumn{2}{c}{$\mathbf{1 9 9 9}$} \\
\cline { 2 - 3 } \cline { 6 - 7 } & $\mathbf{N}^{\mathbf{0}}$ (miles) & \% & & $\mathbf{N}^{\circ}$ & (miles) & \% \\
\hline Consumo diario & 291 & 1,1 & & 110 & 0,4 \\
1-6 días semana & 456 & 1,7 & & 351 & 1,3 \\
1-3 días mes (mensuales) & 649 & 2,5 & & 605 & 2,2 \\
Bimensuales y/o anuales & 3.542 & 13,4 & & 4.014 & 14,7 \\
No se han emborrachado & 14.812 & 56,1 & & 15.004 & 55,0 \\
Abstemios+ & & & & & \\
No han bebido últimos 12 meses + Nc & 6.642 & 25,2 & & 7.197 & 26,4 \\
Totales & 26.397 & 100,0 & & 27.285 & 100,0 \\
\hline
\end{tabular}

Fuente: Encuestas Domiciliarias sobre Drogas 1997, 1999. DGPNSD. Observatorio Español sobre Drogas.

El perfil de las personas que se emborrachan diariamente sigue correspondiéndose con el de los varones adultos (los hombres registran prevalencias cuatro veces superiores a las mujeres), si bien se observa cierto rejuvenecimiento en este tipo de bebedores. Si en 1997 las prevalencias más altas de borracheras diarias se localizaban en ambos sexos en el grupo de 40-65 años, en 1999 las prevalencias se han desplazado hacia grupos más jóvenes: de 30-34 años en los hombres y de 35-39 en las mujeres, en ambos casos seguidos de los de 40-65 años ${ }^{3}$. Por su parte, las borracheras con una frecuencia de 1-2 días a la semana se 
concentran en el grupo de 20-24 años, sin diferencias relevantes entre los sexos.

\section{ACTITUDES ANTE EL CONSUMO DE ALCOHOL}

Las actitudes de los ciudadanos ante las distintas drogas condicionan los hábitos de consumo, de tal modo que no se entendería la importante penetración que el alcohol tiene en la sociedad española sin la existencia de una actitud tolerante ante su consumo. No obstante, es preciso señalar que, aunque el riesgo atribuido al consumo de bebidas alcohólicas continúa siendo bajo, en los últimos años ha crecido la sensibilización respecto a los problemas que comporta el mismo. Existen diferencias relevantes en las actitudes respecto al alcohol, según se trate de consumos diarios o de fin de semana y en función de la edad y el sexo de los encuestados.

a) Actitudes ante el consumo de alcohol en el fin de semana
La mitad de la población española consideraba en 1999 que consumir 5 cañas o copas durante el fin de semana suponía pocos o ningún problema para la salud, mientras que un $48^{\prime} 3 \%$ atribuye a esta conducta bastantes o muchos problemas. Con respecto a 1995 se habría producido un notable cambio, consistente en el hecho de que un $11,5 \%$ de la población habría cambiado su actitud y habría pasado a considerar esta conducta como problemática (Figura 3). El riesgo atribuido alcohol es mayor entre las mujeres (56'5\%) que entre los hombres (41'7\%).

Existen importantes divergencias en las opiniones respecto al consumo de alcohol en función de la edad de los entrevistados (Tabla 14), constatándose que la mayor atribución de peligrosidad se registra entre la población adulta (de 40-65 años) y los menores de 19 años. Por el contrario, los grupos de edad jóvenes (20-34 años), quienes participan mayoritariamente del hábito de ingerir alcohol en el fin de semana, son los que atribuyen una menor peligrosidad a esta conducta. Entre 1995 y 1999 el riesgo atribuido al con-

\section{Figura 3. Evolución de las actitudes ante el consumo de 5 cañas o copas durante}

el fin de semana. España 1995-1999.

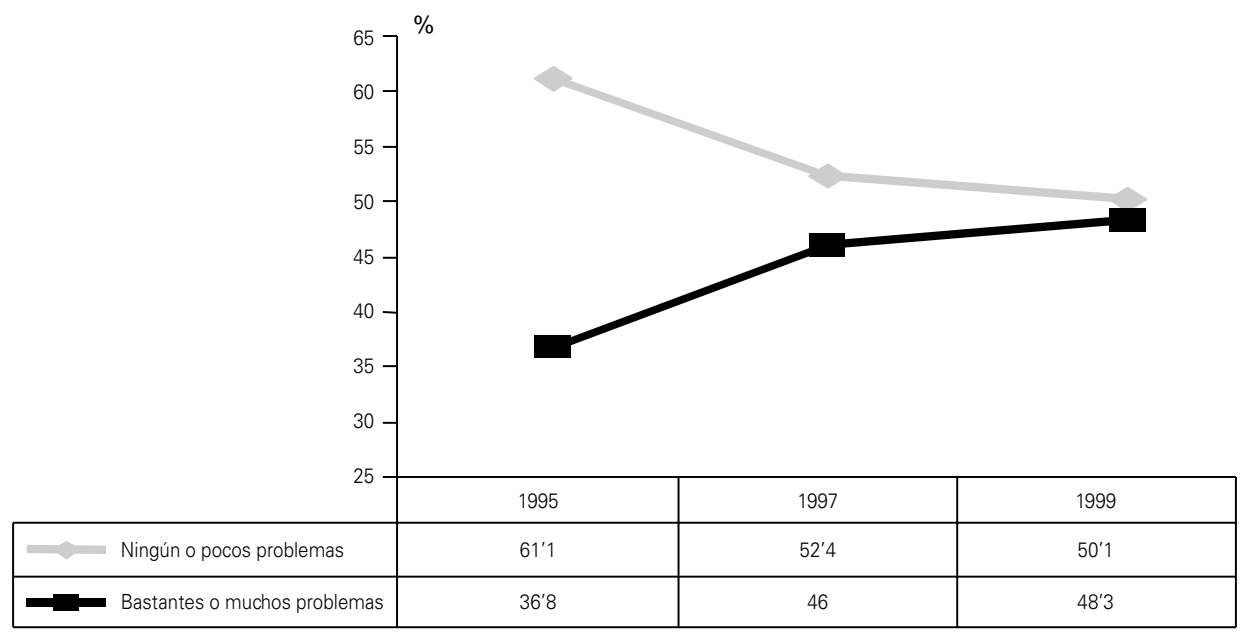

Variaciones 1995/1999. Ninguno o pocos problemas $-11 \%$. Bastantes o muchos problemas $+11^{\prime} 5 \%$.

Fuente: DGPNSD. Observatorio Español sobre Drogas (OED). Encuesta Domiciliaria sobre Drogas 1995, 1997 y 1999 
sumo de alcohol en el fin de semana se habría incrementado en ambos sexos y en todos los grupos de edad, de forma más acu- sada entre los hombres de 25-29 y de 40-65 años y las mujeres de 20-24 años.

Tabla 14. Evolución de las actitudes ante el consumo de cinco cañas y/o copas en el fin de semana por sexo y grupo de edad (porcentaje). España, 1995-1999.

\begin{tabular}{|c|c|c|c|c|c|c|c|c|c|c|c|c|}
\hline \multirow{3}{*}{$\begin{array}{l}\text { Grupo } \\
\text { de edad } \\
\text { (años) }\end{array}$} & \multicolumn{6}{|c|}{ Ninguno + Pocos problemas } & \multicolumn{6}{|c|}{ Bastantes + Muchos problemas } \\
\hline & \multicolumn{2}{|c|}{1995} & \multicolumn{2}{|c|}{1997} & \multicolumn{2}{|c|}{1999} & \multicolumn{2}{|c|}{1995} & \multicolumn{2}{|c|}{1997} & \multicolumn{2}{|c|}{1999} \\
\hline & Hom. & Muj. & lom. & Muj. & lom. & Muj. & Hom. & Muj. & Hom. & Muj. & Hom. & Muj. \\
\hline & & & & & & & & & & & & \\
\hline & & & & & & & & & & & & \\
\hline & & & & & & & & & & & & \\
\hline & & & & & & & & & & & & 45,5 \\
\hline & & & & & & & & & $38,0 \%$ & & $34,2 \%$ & $50,9 \%$ \\
\hline $40-65$ & $65,6 \%$ & $45,2 \%$ & $56,6 \%$ & $35,5 \%$ & $52,3 \%$ & & $34,4 \%$ & $54,8 \%$ & $43,5 \%$ & $64,5 \%$ & $47,7 \%$ & $66,5 \%$ \\
\hline
\end{tabular}

Fuente: Encuestas Domiciliarias sobre Drogas 1995,1997, 1999. DGPNSD. Observatorio Español sobre Drogas.

b) Actitudes ante el consumo diario de alcohol

La sensibilidad frente a los problemas derivados del consumo diario (tomar 5 cañas o copas) de bebidas alcohólicas es elevada, como lo confirma el que casi 9 de cada 10 encuestados considere que esta conducta puede causar bastantes y muchos problemas, habiendo aumentado progresivamente desde 1995 (Figura 4). Entre 1995 y 1999 un $3,7 \%$ de los encuestados habría cambiado su

Figura 4. Evolución de las actitudes ante el consumo de 5 cañas o copas a diario (porcentaje). España 1995-1999.

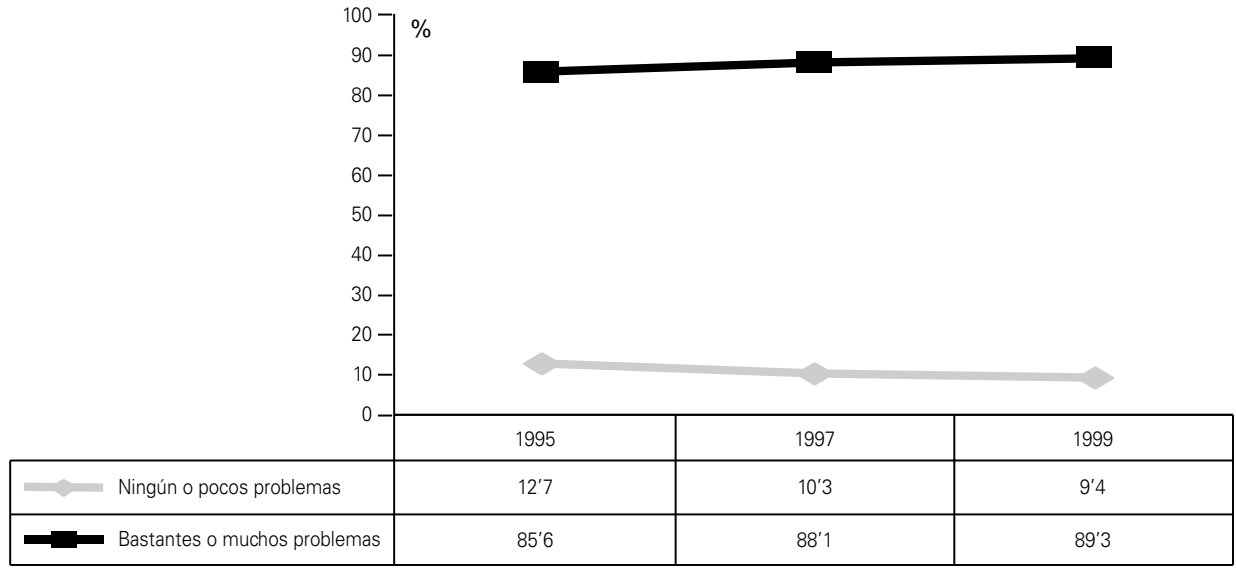

Variaciones 1995/1999. Ninguno o pocos problemas $-3^{\prime} 3 \%$. Bastantes o muchos problemas $+3^{\prime} 7 \%$. 
opinión sobre la inocuidad de consumir alcohol diariamente, habiendo pasado a considerar esta conducta como capaz de crear bastantes o muchos problemas.

Las mujeres atribuyen en mayor proporción bastantes o muchos problemas al consumo diario de alcohol que los hombres. Por otro lado, en 1999, al igual que en 1995, la mayor atribu- ción de problemas se produce en el grupo de 15-19 años (donde son menores las prevalencias de consumo diario) y entre las mujeres adultas (40-65 años). Entre 1995-99 todos los grupos de edad y sexo habrían visto incrementado el riesgo atribuido al consumo diario de alcohol, aunque fue el grupo de 30 a 65 años (Tabla 15) quien lo hizo de modo más intenso.

Tabla 15. Evolución de las actitudes ante el consumo diario de cinco cañas y/o copas por sexo y grupo de edad (porcentaje). España, 1995-1999.

\begin{tabular}{|c|c|c|c|c|c|c|c|c|c|c|c|c|}
\hline \multirow{3}{*}{$\begin{array}{l}\text { Grupo } \\
\text { de edad } \\
\text { (años) }\end{array}$} & \multicolumn{6}{|c|}{ Ninguno + Pocos problemas } & \multicolumn{6}{|c|}{ Bastantes + Muchos problemas } \\
\hline & \multicolumn{2}{|c|}{1995} & \multicolumn{2}{|c|}{1997} & \multicolumn{2}{|c|}{1999} & \multicolumn{2}{|c|}{1995} & \multicolumn{2}{|c|}{1997} & \multicolumn{2}{|c|}{1999} \\
\hline & Hom. & Muj. & Hom. & Muj. & Hom. & Muj. & Hom. & Muj. & Hom. & Muj. & Hom. & Muj. \\
\hline & & 6,5 & & & & & & & & & & 95,4 \\
\hline & & 8,4 & & & & & & & & & & \\
\hline . & & 9,8 & & 8,7 & & & & & & & & \\
\hline & & 10,7 & $15,6 \%$ & 7,4 & & & & & & & & 92,6 \\
\hline & & 8,9 & $17,4 \%$ & 7,6 & & 8,1 & & & & & & \\
\hline $0-65$ & $21,4 \%$ & $5,8 \%$ & $16,6 \%$ & $5,0 \%$ & $12,5 \%$ & $4,2 \%$ & $78,6 \%$ & $94,2 \%$ & $83,4 \%$ & $95,0 \%$ & $87,5 \%$ & $95,8 \%$ \\
\hline
\end{tabular}

Fuente: Encuesta Domiciliarias sobre Drogas 1995, 1997, 1999. DGPNSD. Observatorio Español sobre Drogas.

Las opiniones y actitudes frente al alcohol están claramente relacionadas por el nivel de consumo de los encuestados. La prevalencia de consumo abusivo de alcohol (bebedores altos + excesivos + gran riesgo) en fin de semana es mayor entre quienes opinan que consumir 5 cañas-copas en ese período no provoca problemas (del 10\%), frente a quienes opinan que este tipo de consumo puede generar bastantes o muchos problemas $(4,5 \%)$. Una situación similar se produce respecto a las opiniones sobre el consumo diario de alcohol, observándose que entre quienes opinan que el mismo no provoca problemas se registran prevalencias de consumo abusivo de alcohol del $12,4 \%$, frente al 5 ' $2 \%$ de quienes consideran que puede provocar bastantes o muchos problemas.

\section{EVOLUCIÓN DE LA DEMANDA DE BEBI- DAS ALCOHÓLICAS}

Los datos facilitados por el Panel del Consumo Alimentario, que elabora desde 1987 el
Ministerio de Agricultura, Pesca y Alimentación, referidos a la demanda directa y al gasto realizado por los hogares, establecimientos de hostelería-restauración e instituciones en la adquisición de distintas bebidas alcohólicas, permiten analizar la evolución de los consumos de bebidas alcohólicas desde la perspectiva de la demanda.

El volumen total de las compras de bebidas alcohólicas realizadas en 1999 fue de 3.809,1 millones de litros, situándose las compras per cápita en 95,3 litros. La evolución registrada por las compras de bebidas alcohólicas entre 1995 y 1999 indica que las mismas se redujeron globalmente en un $4^{\prime} 2 \%$, mientras que en este período las compras per cápita descendieron un 5'8\% (Tabla 16). La evolución de las compras de bebidas alcohólicas es congruente con las tendencias observadas en los hábitos de consumo.

\section{MORTALIDAD ASOCIADA AL CONSU- MO DE ALCOHOL}


Tabla 16. Evolución del volumen total de las compras realizadas dentro y fuera del hogar en bebidas alcohólicas. España, 1995-1999 (litros)

\begin{tabular}{|c|c|c|c|c|c|c|c|c|c|c|}
\hline \multicolumn{2}{|c|}{1995} & \multicolumn{2}{|c|}{1996} & \multicolumn{2}{|c|}{1997} & \multicolumn{3}{|c|}{1998} & \multicolumn{2}{|c|}{1999} \\
\hline $\begin{array}{c}\text { Millones } \\
\text { litros }\end{array}$ & $\begin{array}{l}\text { Litros per } \\
\text { cápita }\end{array}$ & $\begin{array}{l}\text { Millones } \\
\text { litros }\end{array}$ & $\begin{array}{l}\text { Litros per } \\
\text { cápita }\end{array}$ & $\begin{array}{l}\text { Millones } \\
\text { litros }\end{array}$ & $\begin{array}{l}\text { Litros per } \\
\text { cápita }\end{array}$ & $\begin{array}{c}\text { Millones } \\
\text { litros }\end{array}$ & & $\begin{array}{l}\text { per } \\
\text { ita }\end{array}$ & $\begin{array}{l}\text { Millones } \\
\text { litros }\end{array}$ & $\begin{array}{l}\text { Litros per } \\
\text { cápita }\end{array}$ \\
\hline $1.200,5$ & 30,6 & $1.298,2$ & 33,0 & $1.392,4$ & 35,0 & 1.41 & 4,7 & 35,6 & $1.371,7$ & 34,31 \\
\hline $2.508,0$ & 63,8 & $2.365,9$ & 60,2 & $2.132,2$ & 53,7 & 2.15 & 3,6 & 54,1 & $2.196,6$ & 54,95 \\
\hline 48,4 & 1,2 & 56,1 & 1,4 & 69,9 & 1,8 & 71 & & 1,8 & 76,96 & 1,93 \\
\hline 217,9 & 5,5 & 205,6 & 5,2 & 178,5 & 4,5 & 173 & & 4,4 & 163,9 & 4,10 \\
\hline $3.974,8$ & 101,1 & $3.925,8$ & 99,8 & $3.773,0$ & 95,0 & 3.81 & 3,5 & 95,9 & 3809,1 & 95,29 \\
\hline
\end{tabular}

Fuente: Panel del Consumo Alimentario 1995, 1996, 1997, 1998, 1999. Ministerio de Agricultura, Pesca y Alimentación.

\subsection{Mortalidad atribuible al consumo de alcohol}

Las estimaciones sobre la mortalidad asociada al consumo de alcohol en España realizadas por la Dirección General de Salud Pública y Consumo ( $\mathrm{M}^{\circ}$ de Sanidad y Consumo), basadas en la utilización del sistema de cálculo propuesto por los Centers for Disease Control (CDC) de los Estados Unidos (Alcohol related Mortality and Years of Potential Life Lost United States 1987,1990), confirman el importantísimo impacto sanitario que tiene el consumo de bebidas alcohólicas.

Sobre la base de la "Estadística de Defunciones según la causa de la Muerte 1997", la última publicada por el Instituto Nacional de Estadística, se ha estimado la mortalidad relacionada con el alcohol en España en el año 1997 en 12.032 fallecimientos, lo que representaría el 3,4\% del total de las defunciones registradas en el país (Tabla 17). La cirrosis y otras enfermedades crónicas del hígado son la primera causa de muerte atribuible al alcohol, seguida de los accidentes de circulación. Con respecto a 1995 se habría producido una ligera reducción del número de fallecimientos atribuibles al alcohol (del 2\%), tendencia coherente con la reducción observada en los consumos abusivos de alcohol.

La reducción en la mortalidad no es homogénea para todos los factores o causas atribuibles, puesto que el número de fallecimien- tos registrados en accidentes de tráfico ha crecido levemente (conviene tener presente que los accidentes de vehículos a motor constituyen la primera causa de defunción entre las mujeres de 1-34 años y entre los hombres de 1-24 años). Con independencia de la edad, las tasas específicas de mortalidad son siempre más altas en los hombres que en mujeres, registrándose en ambos colectivos un especial riesgo entre los 15 y 24 años. Esta distribución, similar a la observada en otros países, sitúa a estos jóvenes, y en especial a los varones, como el grupo de mayor riesgo de sufrir una lesión fatal por accidente de tráfico.

Algunos estudios ${ }^{7}$ atribuían en 1990 al consumo de alcohol 194.868 años de vida perdidos (154.714 en los hombres y 40.154 en las mujeres), destacando el hecho de que los accidentes no intencionales supusieron como media el $49^{\prime} 1 \%$ de los años de vida perdidos por el alcohol entre 1981 y 1990, de los que casi la mitad se corresponderían con accidentes de vehículos a motor.

\subsection{Muertes en accidentes de tráfico rela- cionadas con el consumo de alcohol}

El análisis que desde 1998 realiza periódicamente el Instituto de Toxicología de amplias muestras de cadáveres de conductores y peatones fallecidos en accidentes de 


\section{Tabla 17. Mortalidad atribuible al alcohol según causa de muerte (número absoluto y porcentaje). España, 1997.}

\begin{tabular}{lccc}
\hline Causa de muerte & $\begin{array}{c}\text { Número } \\
\text { absoluto }\end{array}$ & $\begin{array}{c}\text { Fracción } \\
\text { atribuible (\%) }\end{array}$ & $\begin{array}{c}\mathbf{N}^{\circ} \text { muertes } \\
\text { atribuibles }\end{array}$ \\
\hline Cirrosis y otras enfermedades crónicas del hígado (E-571) & 6.423 & 70 & 4.496 \\
Accidentes de tráfico de vehículos de motor (E 810-819) & 5.790 & 40 & 2.316 \\
Pancreatitis aguda (E-77.0) & 1.105 & 42 & 464 \\
Psicosis alcohólica (E-291) & 56 & 100 & 56 \\
Síndrome de dependencia alcohólica (E-303) & 199 & 100 & 199 \\
Abuso de alcohol (E-305.0) & 13 & 100 & 13 \\
Envenenamiento accidental por alcohol, no clasificado & & & \\
en otra parte (E-860) & 15 & 100 & 15 \\
Suicidios y lesiones autoinfligidas (E-950-959) & 3.373 & 25 & 843 \\
Cardiomiopatía alcohólica (E-425.5) & 30 & 100 & 30 \\
Gastritis alcohólica (E-535.3) & 3 & 100 & 3 \\
Polineuropatía alcohólica (E-357.5) & 4 & 100 & 4 \\
Pancreatitis crónica (E-577.1) & 45 & 60 & 27 \\
T. maligno labio, cavidad oral, faringe (E 140-149) & 2.268 & 50 & 1.134 \\
T. maligno esófago (E-150) & 1.762 & 75 & 1.321 \\
T. maligno laringe (E-161) & 1.909 & 50 & 954 \\
Homicidio (E 960-969) & 342 & 46 & 157 \\
Total & 23.337 & & 12.032 \\
\hline
\end{tabular}

Fuente: Defunciones según la causa de muerte 1997. INE 2000. Elaboración: Dirección General de Salud Pública y Consumo ( $\mathrm{M}^{\circ}$ de Sanidad y Consumo).

tráfico, en virtud del convenio de colaboración suscrito entre la Delegación del Gobierno para el Plan Nacional Sobre Drogas, permite confirmar la estrecha relación existente entre el consumo de alcohol y otras drogas de abuso con las muertes de conductores y peatones producidas en accidentes de tráfico. De un total de 1.191 cadáveres analizados de conductores fallecidos en accidentes de tráfico durante 1999, en el $37^{\prime} 4 \%$ se detectó la presencia de alcohol, bien sólo o junto con psicofármacos y otras drogas de abuso. Pero sin duda el dato más relevante lo constituye el que en el $29^{\prime} 8 \%$ de los cadáveres se identificaron niveles de alcoholemia superiores a 0’5grs./litro, la tasa máxima legalmente permitida.

A pesar de lo preocupante de estas cifras, en el período 1998-99 se habría registrado un significativo descenso de la presencia de alcohol y otras drogas en los conductores fallecidos en accidentes de tráfico. De hecho la presencia exclusiva de alcohol, pasó de detectarse en el $43^{\prime} 2 \%$ de los fallecidos en 1998 al 37'4\% en 1999 (Tabla 18).

Por otra parte, en el $35^{\prime} 4 \%$ de los cadáveres analizados durante 1999 de peatones atropellados se identificó la presencia de alcohol, debiéndose destacar que uno de cada cuatro peatones atropellados (el $25^{\prime} 8 \%$ ) presentaba tasas de alcoholemia superiores a 0’5 grs./litro.

Como han puesto de manifiesto diversos estudios $^{8}$ el impacto del consumo de bebidas alcohólicas en los accidentes de tráfico en los fines de semana es especialmente notable. El grueso de los accidentes mortales de tráfico, se produce en las noches del fin de semana y afecta principalmente a jóvenes. De hecho una de cada tres muertes de jóvenes menores de 30 años se produce a consecuencia de los accidentes de tráfico. 
Tabla 18. Sustancias psicoactivas detectadas en los análisis toxicológicos de las muestras biológicas de los conductores de vehículos fallecidos en accidentes de tráfico (número y porcentaje). España 1998-1999.

\begin{tabular}{lccccc}
\hline & \multicolumn{2}{c}{1998} & & \multicolumn{2}{c}{1999} \\
\cline { 2 - 3 } \cline { 6 - 7 } & Número & Porcentajes & & Número & Porcentajes \\
\hline Alcohol etílico & 471 & $43,2 \%$ & & 446 & $37,4 \%$ \\
Opiáceos* & 45 & $4,1 \%$ & & 29 & $2,4 \%$ \\
Cocaína & 51 & $4,6 \%$ & & 62 & $5,2 \%$ \\
Anfetamina & 15 & $1,4 \%$ & & 6 & $0,5 \%$ \\
MDMA y Derivados & 4 & $0,5 \%$ & & 5 & $0,4 \%$ \\
Barbitúricos & 0 & $0,0 \%$ & & 1 & $0,1 \%$ \\
Benzodiazepinas & 32 & $2,9 \%$ & & 41 & $3,4 \%$ \\
Antipsicóticos & 1 & $0,1 \%$ & & 0 & $0,0 \%$ \\
Cannabis & 26 & $2,4 \%$ & & 28 & $2,5 \%$ \\
Antidepresivos & 8 & $0,7 \%$ & & 6 & $0,5 \%$ \\
Total análisis & 1.090 & & & 1.191 & \\
\hline
\end{tabular}

(*) En este grupo se ha incluido la metadona.

Fuente: Instituto Nacional de Toxicología (Departamentos de Barcelona, Madrid, Sevilla y La Laguna).

\section{REFERENCIAS BIBLIOGRÁFICAS}

(1) SANCHEZ PARDO, L. Situación actual y evolución de los consumos de drogas ilícitas en España. Trastornos Adictivos, 2001, vol. 3, $n^{\circ}$ 2, pp 85-94.

(2) MEGÍAS, E. et al. La percepción social de los problemas de drogas en España. Madrid: Fundación de Ayuda Contra la Drogadicción, 2000.

(3) DELEGACIÓN DEL GOBIERNO PARA EL PLAN NACIONAL SOBRE DROGAS. Informe $\mathbf{N}^{\circ} \mathbf{4}$ del Observatorio Español sobre Drogas. Madrid: Delegación del Gobierno para el Plan Nacional sobre Drogas, 2001.

(4) DELEGACIÓN DEL GOBIERNO PARA EL PLAN NACIONAL SOBRE DROGAS. Encuesta sobre drogas a población escolar, 2000. Madrid:
Delegación del Gobierno para el Plan Nacional sobre Drogas, 2001.

(5) ORGANIZACIÓN MUNDIAL DE LA SALUD. Alcohol Policy Profiles in the European Region of the World Hraltth Organization. WHO Regional Office for Europe, 2001.

(6) DELEGACIÓN DEL GOBIERNO PARA EL PLAN NACIONAL SOBRE DROGAS. Informe $\mathbf{N}^{\circ} \mathbf{2}$ del Observatorio Español sobre Drogas. Madrid: Delegación del Gobierno para el Plan Nacional sobre Drogas, 2000.

(7) PRADA, C. et al. Mortalidad relacionada con el consumo de alcohol. Gaceta Sanitaria, 1996, vol. 10, pp 161-168.

(8) MONDON, S. et al. Estudio epidemiológico del consumo de bebidas alcohólicas en accidentes de tráfico en los fines de semana. Adicciones, 1997, vol. 9 n³, pp 391-403. 